\title{
Prognostic effect of programmed death- ligand 1 (PD-L1) in ovarian cancer: a systematic review, meta-analysis and bioinformatics study
}

\author{
Lin Wang(D
}

\begin{abstract}
Background: The expression of PD-L1 has been reported in ovarian cancer. However, the prognostic role of PD-L1 expression in ovarian carcinoma remained controversial. This study was performed to assess the prognostic value of PD-L1 expression on ovarian cancer.

Methods: The PubMed, Embase, EBSCO, and Cochrane Library databases were searched to identify available publications. The pooled odds ratio (OR) or hazard ratios (HRs: multivariate analysis) with their $95 \%$ confidence intervals ( $95 \% \mathrm{Cls}$ ) were calculated in this analysis. A bioinformatics study based on The Cancer Genome Atlas (TCGA) sequencing and microarray datasets was used to further validate the results of PD-L1 mRNA expression. Kaplan-Meier (KM) survival curves were performed to evaluate the prognostic effect of PD-L1 mRNA expression.

Results: Twelve studies with 1630 ovarian cancers regarding PD-L1 immunohistochemical expression were identified. Meta-analysis showed that PD-L1 protein expression was not associated with tumor grade, clinical stage, lymph node status, tumor histology, overall survival (OS), and progression-free survival (PFS). TCGA data showed no association between PD-L1 mRNA expression and ovarian cancer. Further validation using microarray data suggested that no association between PD-L1 mRNA expression and OS was found in large independent patient cohorts (1310 cases). PD-L1 mRNA expression was significantly linked to worse PFS in 1228 patients with ovarian cancer (227458_at: $H R=1.55,95 \% \mathrm{Cl}=1.28-1.88, P<0.001$; 223834_at: $\mathrm{HR}=1.41$, $95 \% \mathrm{Cl}=1.14-1.75, P=0.0015)$.

Conclusions: Meta-analysis showed that PD-L1 may not be a prognostic factor for ovarian cancer. But a bioinformatics study showed that PD-L1 expression was significantly associated with worse PFS of ovarian cancer. More clinical studies are needed to further validate these findings.
\end{abstract}

Keywords: PD-L1, Ovarian cancer, Prognosis, Therapy

\section{Introduction}

Ovarian cancer is the second most common human gynecological malignancy and the most deadly gynecological malignancy among women [1]. According to global statistics, approximately 238,700 new cases were clinically diagnosed with ovarian carcinoma, and it killed 151,900 cases worldwide in 2008 [1]. Serous histology is the most common ovarian cancer, and other types consist

Correspondence: wanglinfb@zju.edu.cn

Women's Hospital, School of Medicine, Zhejiang University, Hangzhou 310006, China of mucinous, clear cell and endometrioid carcinomas etc. [2]. Early stage patients with ovarian carcinoma generally have a favorable prognosis. However, most patients are diagnosed with advanced stages of this disease (stage 3-4), with a five-year survival rate of less than $20 \%[3,4]$.

Programmed cell death receptor 1 (PD-1) belongs to the B7-CD28 family of costimulatory receptors and is expressed on the surface of T, B, and Natural killer (NK) cells that play key roles their activation and apoptosis [5, 6]. Programmed cell death-ligand 1 (PD-L1), also known as cluster of differentiation 274 (CD274) or B7-H1, is 
one of the PD-1 ligands and is expressed in on tumor cells and immune cells. PD-L1 is considered to be a crucial immunological escape mechanism that results in tumor cell growth, proliferation and metastasis [7-9]. Immune checkpoint blockade of PD-L1 with monoclonal antibody has shown promising approaches for improving survival rates of cancer patients [10]. PD-L1 expression has been reported to be associated with poor prognosis in many human cancers [11]. PD-L1 is also expressed in ovarian cancer. However, data regarding the prognostic effect of PD-L1 expression in ovarian cancer are limited, and some findings remain controversial. Hamanishi 2007 et al. reported that PD-L1 expression was correlated with poor overall survival in ovarian cancer [12]. But no association between PD-L1 expression and overall survival was found by Mills 2018 et al. [13].

To address the above-described issue, based on available publications, TCGA sequencing and microarray datasets, we conducted a systematic analysis to evaluate the relationship of PD-L1 expression with clinicopathological characteristics of ovarian cancer and the prognostic effect of PD-L1 expression.

\section{Materials and methods}

\section{Systematic review}

The PubMed, Embase, EBSCO, and Cochrane Library databases were systematically searched to identify all available studies using the following key words and search terms updated to September 16, 2018: 'programmed cell death protein 1 OR PD-1 OR programmed cell death ligand 1 OR programmed cell death-ligand 1 OR CD274 OR B7-H1 OR PD-L1', 'ovarian OR ovary', 'cancer OR carcinoma OR tumor OR neoplasm'. In addition, a manual search from reference lists of all eligible studies was also conducted to get additional articles. This systematic review was conducted in accordance with the preferred reporting items for systematic reviews and meta-analyses (PRISMA) statement criteria [14].

The following inclusion criteria for the eligible publications were applied in this systematic review and meta-analysis: 1) the patients had a diagnosis of ovarian cancer based on histopathological examination; 2) cohort studies on the expression of PD-L1 using immunohistochemical (IHC) staining; 3) studies provided data to evaluate the relationship between PD-L1 expression and clinicopathological features of ovarian cancer patients (tumor histology, cancer grade, clinical stage, and lymph node status etc.); 4) studies reported sufficient information between PD-L1 expression and the prognosis of ovarian cancer using multivariate analysis, such as overall survival (OS) or progression-free survival (PFS). If authors published more than one paper using the overlapping population data, only the paper with more information was included in the current analysis.
The primary endpoint was OS, which was recorded as the time from the study enrollment to the date of death due to any cause or last follow-up. The secondary endpoint was PFS, which was defined as the time from the study enrollment until the first observed tumor progression or death.

The main exclusion criteria were as follows: 1) reviews, letters, case reports, or conference abstracts; 2) studies on cell lines and animals; 3 ) the detection method was not IHC; 4) studies lacking available data of PD-L1 expression and ovarian cancer, and 5) survival data using univariate analysis.

According to the selection criteria, author independently extracted necessary information from original articles in this systematic review and meta-analysis: first author's surname, publication year, country, ethnic population, IHC staining, the frequency of PD-L1 expression, population size, effects on the clinical prognosis for multivariate analysis, and clinicopathological features such as tumor histology, tumor differentiation, clinical stage, and lymph node status etc..

\section{Bioinformatics study from TCGA sequencing and microarray datasets}

The RNA-sequencing data and corresponding clinical information of patients with ovarian cancer were downloaded from The Cancer Genome Atlas (TCGA) (https://cancergenome.nih.gov/). Finally, 374 cancer patients with the available clinical data were identified. We determined the cut-off value of PD-L1 mRNA expression based on its median value. The Kaplan-Meier plotter tool (http://kmplot.com/analysis/) was also used to further analyze the clinical outcomes of PD-L1 mRNA expression using microarray data in ovarian cancer [29]. In this KM plotter database, PD-L1 mRNA expression data with OS information of 1310 ovarian cancer patients and PFS information of 1228 ovarian cancer patients were obtained.

\section{Statistical analysis}

The overall odds ratios (ORs) with their 95\% confidence intervals (95\% CIs) were calculated for estimating the correlation between PD-L1 expression and the clinicopathological features of ovarian cancer. The combined hazard ratios (HRs) with their 95\% CIs were used to determine the prognostic effect of PD-L1 expression in ovarian cancer. The heterogeneity among the eligible studies was detected using the Cochran's Q test [15]. The random-effects model was used to make the results more reliable in the present study [16]. If substantial heterogeneity was detected for significant results $(P<0.1)$, a sensitivity analysis was performed to determine the influence of the pooled results by omitting an individual study [17]. For the results with greater than four studies, Egger's test 
was used to measure the possible publication bias [18]. The pooled data from meta-analysis were analyzed using the Stata software, version 12.0 (Stata Corp., College Station, TX, USA).

The relationships between PD-L1 mRNA expression and the clinical characteristics were conducted by using the univariate logistic regression analysis. Survival curve was determined by Kaplan-Meier method and log-rank test. The univariate and multivariate Cox analyses were used to evaluate the role of PD-L1 expression on survival if possible. TCGA data were analyzed using R (v. 3.5.1, Institute for Statistics and Mathematics, Vienna, Austria). In the KM plotter database, OS and PFS of patients with ovarian cancer by a Kaplan-Meier survival plot with HR and $\log$-rank $P$ value were determined.

\section{Results}

\section{Systematic review}

According to the above-described inclusion criteria and exclusion criteria, final 12 articles published from 2007 to 2018 [12, 13, 19-28] were identified in the systematic review and meta-analysis (Fig. 1), including 1630 patients with ovarian cancer. PD-L1 protein expression was detected using the IHC method. Nine studies evaluated the association of PD-L1 expression with clinicopathological characteristics of ovarian cancer $[12,13,19$, 20, 22-24, 26, 27]. Ten studies with 1525 ovarian cancer patients evaluated the prognostic role of PD-L1 expression using multivariate analysis $[12,13,19,21-25,27$,
28]. The main characteristics of the eligible studies are shown in Table 1.

The results of meta-analysis demonstrated that PD-L1 expression was not correlated with tumor grade $(\mathrm{OR}=1.63$, 95\% CI $=0.90-2.96, P=0.109, \mathrm{n}=$ three studies with 640 ovarian cancer patients) and clinical stage $(\mathrm{OR}=1.14,95 \%$ $\mathrm{CI}=0.68-1.91, P=0.607, \mathrm{n}=$ nine studies with $1326 \mathrm{pa}-$ tients with ovarian cancer) (Fig. 2). Data of meta-analysis from two studies with 164 ovarian cancer patients showed no association between PD-L1 expression and lymph node status $(\mathrm{OR}=1.43,95 \% \mathrm{CI}=0.68-3.03, P=0.35)$ (Fig. 2). Meta-analysis showed that PD-L1 expression was not associated with tumor histology $(\mathrm{OR}=1.47,95 \% \mathrm{CI}=0.47$ 4.55, $P=0.507$ ) (Fig. 2), including four studies with 880 ovarian cancer patients.

As shown in Table 2, only Zhu 2018 et al. reported that PD-L1 expression was not linked to OS and DFS in 112 ovarian cancer patients [19]. Only Webb 2016 et al. [27] reported that PD-L1 expression was correlated with favorable disease-specific survival $(\mathrm{HR}=0.607,95 \% \mathrm{CI}=$ 0.399-0.925) in 195 patients with serous ovarian cancer. Based on qualitative analysis of DFS and disease-specific survival, more studies are needed to further validate the prognostic effect of PD-L1 expression in DFS and disease-specific survival in the future. Meta-analysis of long-term survival showed that PD-L1 expression was not linked to OS (seven studies with 835 patients: $H R=$ 1.13, 95\% CI $=0.63-2.04, P=0.673$ ) and PFS (five studies with 495 patients: $\mathrm{HR}=1.18,95 \% \mathrm{CI}=0.70-1.98, P=$ 0.532) (Fig. 3).

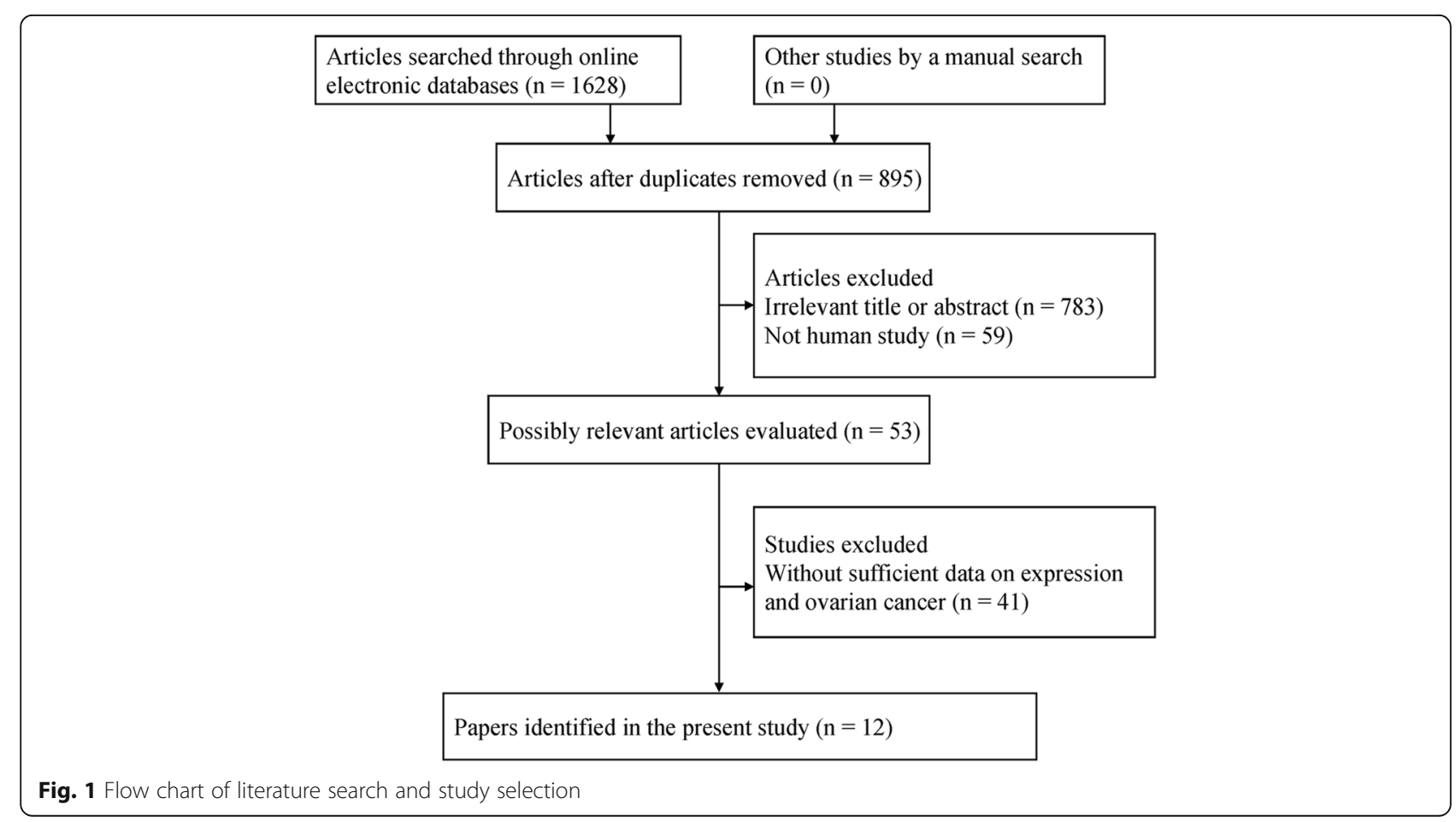




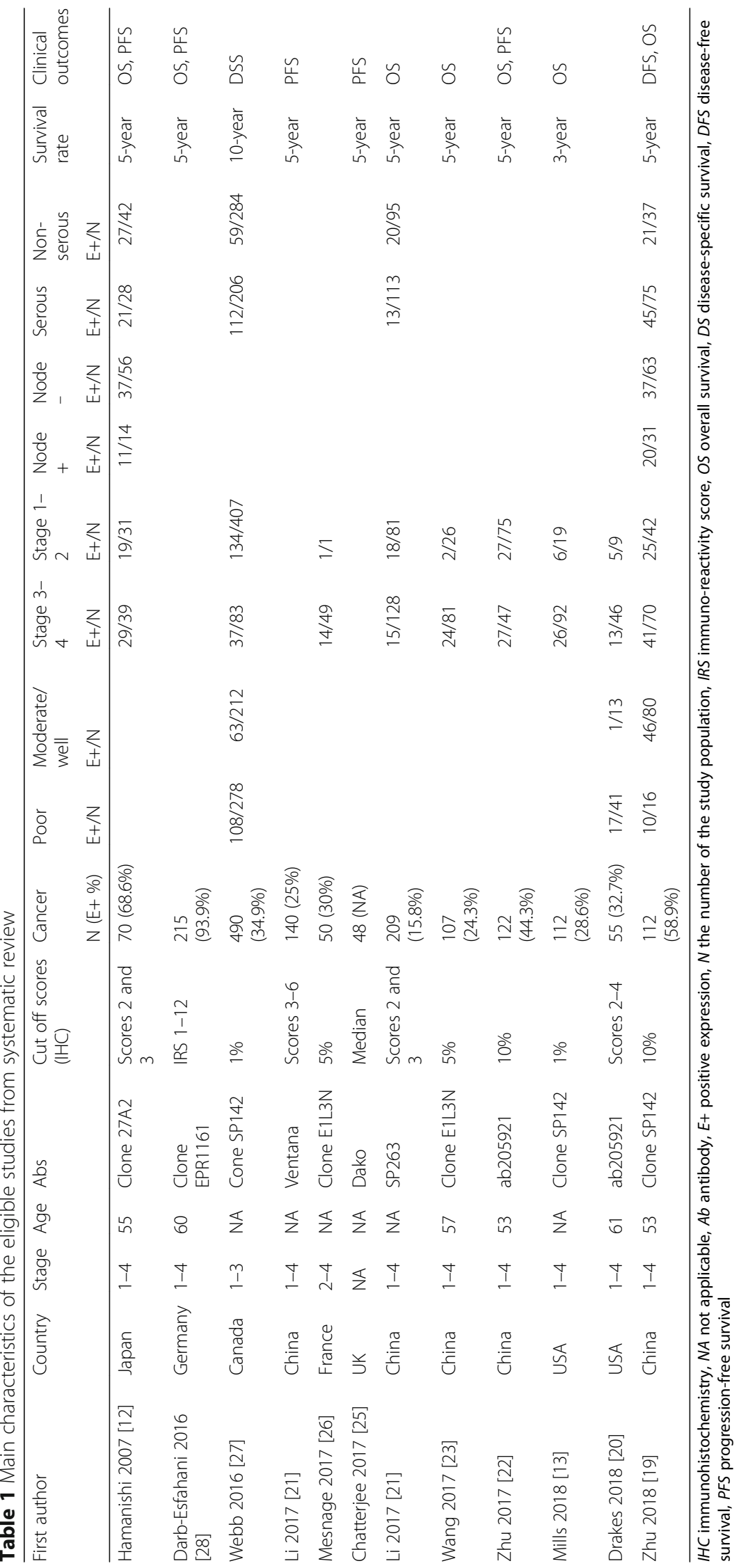




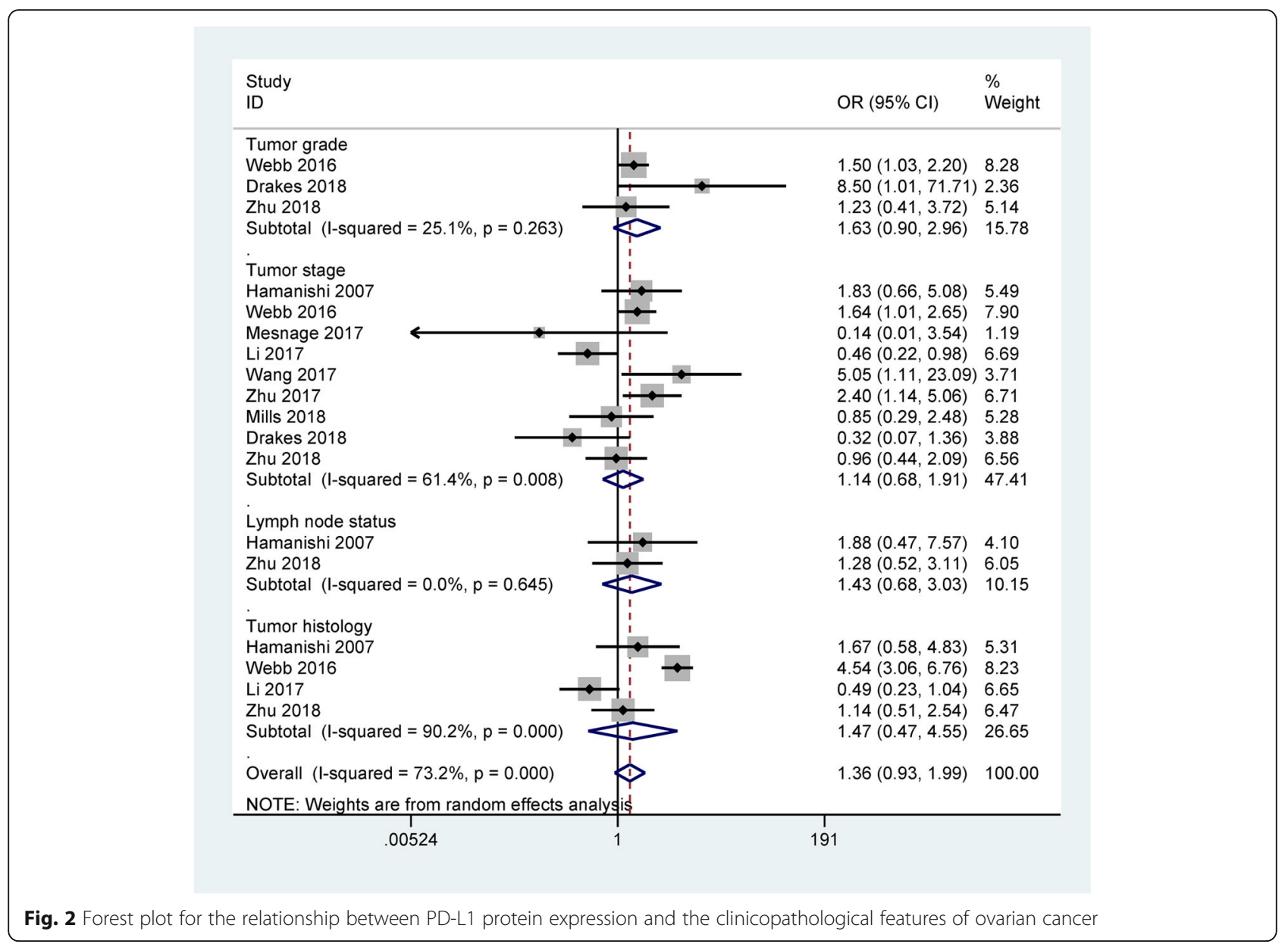

Heterogeneity of meta-analysis was found in relation to tumor stage $(P=0.008)$, when two studies $[20,24]$ were removed, heterogeneity was significantly reduced $(P=0.198)$ and the re-calculated OR was $1.56(95 \% \mathrm{CI}=1.03-2.36, P=$ $0.037)$. Heterogeneity of meta-analysis was observed in relation to tumor histology $(P<0.001)$, when two studies [24, 27] were removed, heterogeneity was obviously decreased $(P=0.578)$. The pooled OR was not significantly changed $(\mathrm{OR}=1.31,95 \% \mathrm{CI}=0.69-2.48, P=0.408)$.

Heterogeneity of meta-analysis was observed between PD-L1 expression and OS $(P=0.001)$. When we deleted three studies $[12,22,28]$, the re-calculated HR was not significantly changed $(\mathrm{HR}=0.92,95 \% \mathrm{CI}=0.58-1.46, P=$ $0.727)$, with no heterogeneity $(P=0.287)$. Heterogeneity of meta-analysis was found between PD-L1 expression and PFS $(P=0.001)$. When we removed two studies $[25,28]$, heterogeneity was significantly decreased $(P=0.678)$ and the re-calculated $\mathrm{HR}$ was significant $(\mathrm{HR}=1.98,95 \% \mathrm{CI}=1.27-$ 3.10, $P=0.003)$.

To examine the potential publication bias, Egger's test was performed in the meta-analysis. The results showed that no publication bias was found between PD-L1 expression and tumor stage, OS, and PFS (all $P$ values > 0.05) (Additional file 1: Figure. S1).

\section{TCGA and microarray datasets}

The available clinical information were identified from TCGA for serous ovarian cancer. The univariate logistic

Table 2 Summary of clinical outcomes from systematic review

\begin{tabular}{llllll}
\hline & Studies & HR with 95\% Cl & $P_{\text {heterogeneity }}$ & $P$ values & N \\
\hline Overall survival & 7 & $1.13(0.63-2.04)$ & 0.001 & 0.673 & 835 \\
Progression-free survival & 5 & $1.18(0.70-1.98)$ & 0.001 & 0.532 & 495 \\
Disease-specific survival & 1 & $0.607(0.399-0.925)$ & NA & 0.02 & 195 \\
Disease-free survival & 1 & NA & NA & $>0.05$ & 112 \\
\hline
\end{tabular}

HR hazard ratio, 95\% Cl 95\% confidence interval, NA not applicable, $N$ the number of the study population 


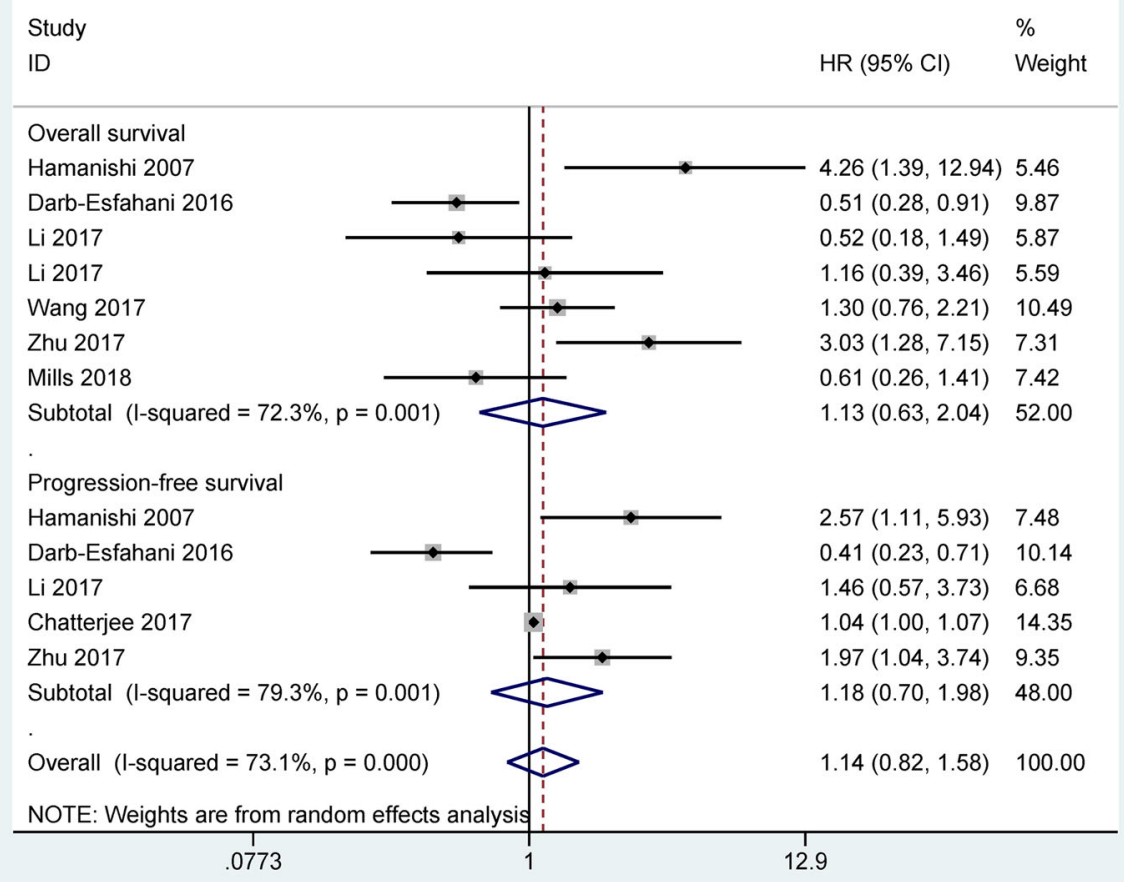

Fig. 3 Forest plot for the association between PD-L1 protein expression and the prognosis

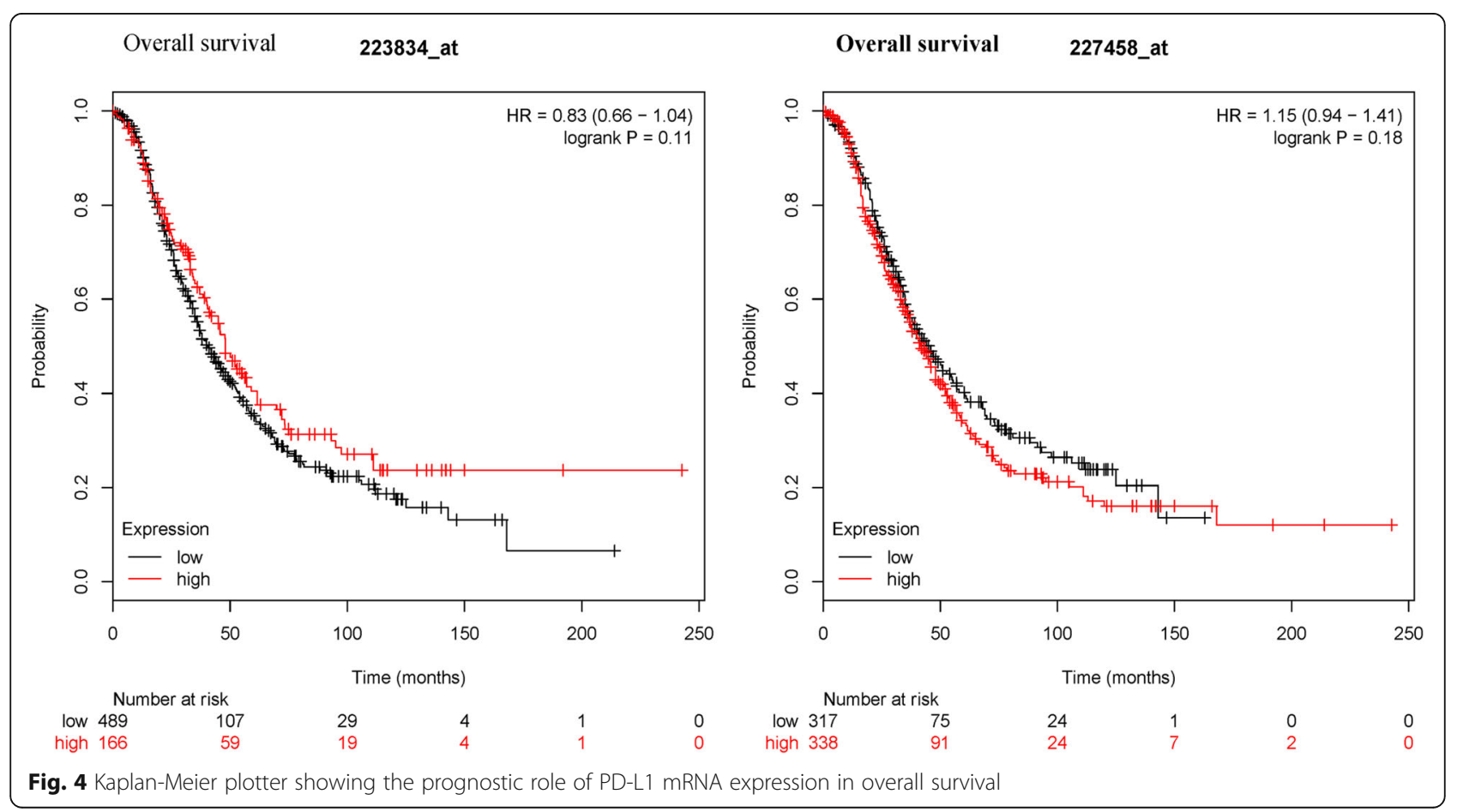


regression analysis showed that PD-L1 mRNA expression was not significantly correlated with the clinicopathological characteristics of patients with ovarian cancer (Additional file 2: Table S1), including tumor residual disease (yes vs. no: $\mathrm{OR}=0.75,95 \% \mathrm{CI}=0.44-1.29$, $P=0.297$ ), cancer status (with tumor vs. tumor free: $\mathrm{OR}$ $=0.94,95 \% \mathrm{CI}=0.57-1.54, P=0.798$ ), venous invasion (positive vs. negative: $\mathrm{OR}=1.8,95 \% \mathrm{CI}=0.81-4.02, P=$ 0.149 ), lymphatic invasion (positive vs. negative: $\mathrm{OR}=$ 1.67, $95 \% \mathrm{CI}=0.83-3.35, P=0.147$ ), tumor grade (poor vs. well or moderate: $\mathrm{OR}=1.34,95 \% \mathrm{CI}=0.7-2.53, P=$ 0.375 ), and clinical stage (stage $3-4$ vs. $1-2$ : $\mathrm{OR}=0.75$, 95\% CI $=0.32-1.76, P=0.511$ ). Kaplan-Meier survival showed that PD-L1 mRNA expression was not associated with OS in 374 patients with ovarian cancer (data not shown).

An online KM plotter database using microarray dataset further showed that PD-L1 mRNA expression was not linked to OS in 1310 patients with ovarian cancer (227458_at: $\quad H R=1.15, \quad 95 \% \quad C I=0.94-1.41, \quad P=0.18$; 223834_at: $\mathrm{HR}=0.83,95 \% \mathrm{CI}=0.66-1.04, P=0.11$ ) (Fig. 4). PD-L1 mRNA expression was significantly correlated with poor PFS in 1228 patients with ovarian cancer (227458_at: $\mathrm{HR}=1.55,95 \% \mathrm{CI}=1.28-1.88, P=7.3 \times 10^{-6}$; 223834_at: $\mathrm{HR}=1.41,95 \% \mathrm{CI}=1.14-1.75, P=0.0015)$ (Fig. 5).

\section{Discussion}

Cancer immunotherapy is a novel approach of cancer treatment targeting the immune checkpoint receptors such as PD-L1. PD-L1 is an important immune regulatory factor and is closely correlated with the immune escape mechanism of cancer cells [30]. PD-L1 upregulation can be motivated by cytokines induced by tumor-infiltrating immune cells, including interferon (IFN), tumor necrosis factor (TNFalpha), interleukin (IL-4), and vascular endothelial growth factor (VEGF) etc. [31-34]. Blockade of the PD-L1 pathway is a promising immune-based treatment [35]. PD-L1 is expressed in many human cancers such as lung cancer [36], breast cancer [37], hepatocellular carcinoma [38], and cervical cancer [39]. PD-L1 expression in ovarian cancer has received great attention in recent years. At present, the association between PD-L1 expression and the prognostic role of ovarian cancer patients remains controversial $[12,13,19,21-25,28]$. Thus, the current study using available articles (this systematic review and meta-analysis), TCGA sequencing, and microarray datasets was conducted to evaluate whether PD-L1 expression was linked to different clinicopathological features and prognostic signature of patients with ovarian carcinoma. This work found that PD-L1 expression was significantly correlated with worse PFS, but was not associated with OS in ovarian cancer, suggesting that PD-L1 may serve as a useful prognostic marker for predicting PFS and serve as a therapeutic target in ovarian cancer.

The relationship between PD-L1 expression and the clinicopathological characteristics of ovarian cancer was analyzed. The results comprising all eligible studies with large populations showed that PD-L1 expression was not associated with tumor grade (poor differentiation vs. well/moderate differentiation, clinical stage (stage 3-4

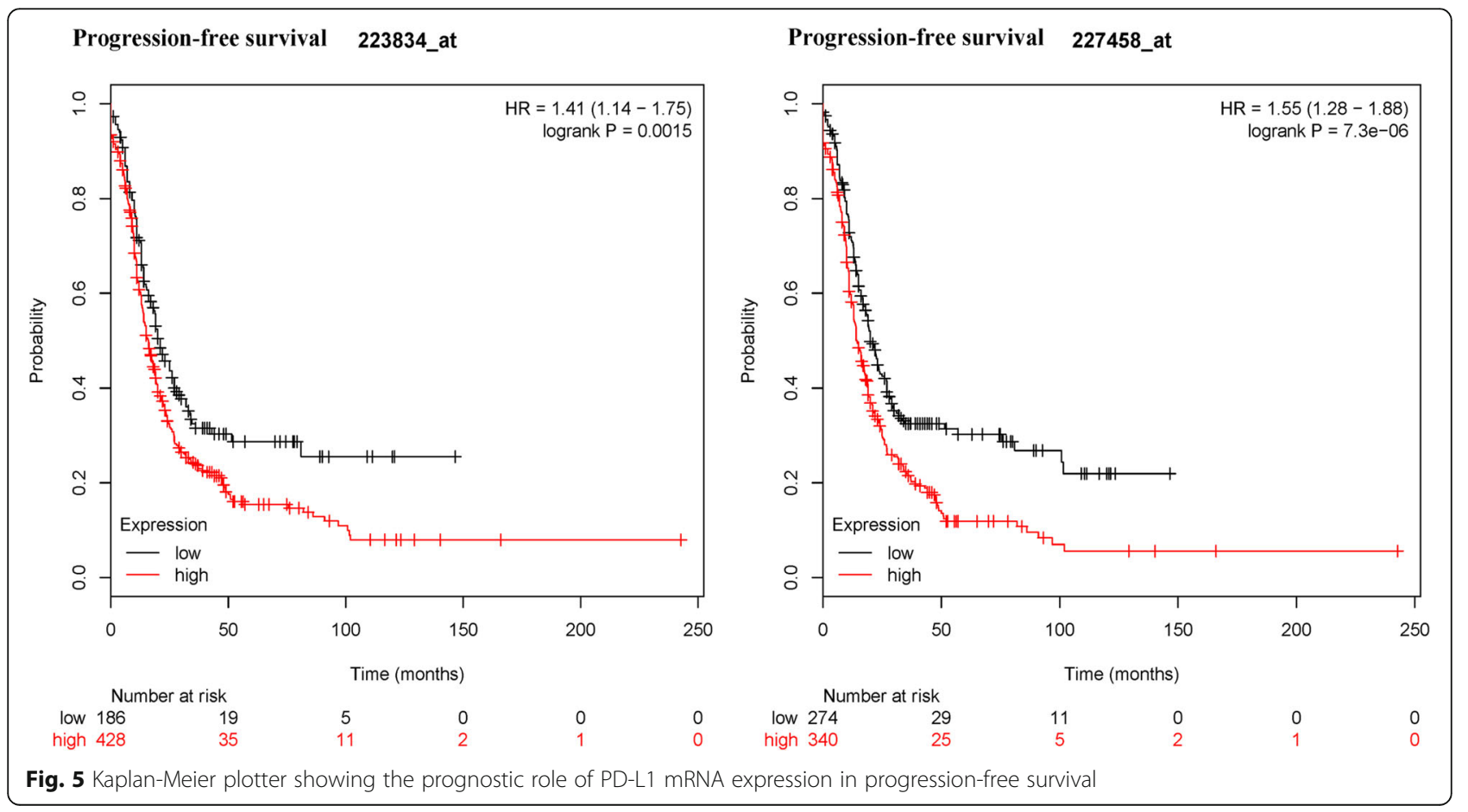


vs. stage 1-2), and lymph node status (positive vs. negative), and tumor histology (serous vs. non-serous carcinoma) in this meta-analysis. Heterogeneity was observed between PD-L1 expression and clinical stage and tumor histology $(P<0.1)$. When we removed two studies $[20$, $24]$ in relation to clinical stage and two studies [24, 27] in relation to tumor histology. The results showed that heterogeneity was significantly reduced $(P>0.1)$, the re-calculated OR was not significantly changed for tumor histology, while the re-calculated OR indicated that PD-L1 expression was correlated with clinical stage $(\mathrm{OR}=1.56, P=0.037)$. The possible reasons of the heterogeneity remained unclear, perhaps because inappropriate conditions of immunohistochemical (IHC) staining may be used, which might lead to the observed bias in the present meta-analysis. Further bioinformatics data from TCGA showed that PD-L1 expression was not significantly associated with tumor residual disease, cancer status, venous invasion, lymphatic invasion, tumor grade, and clinical stage, which further suggested that PD-L1 expression was not correlated with advanced clinicopathological characteristics of patients with ovarian cancer.

Data from the present meta-analysis demonstrated that PD-L1 expression was not linked to the prognosis of ovarian cancer patients in OS and PFS. Heterogeneity was found between PD-L1 expression and the prognosis. When three studies $[12,22,28]$ were removed in OS and two studies $[25,28]$ were removed in PFS. No heterogeneity was measured in OS and PFS. The re-calculated results indicated that PD-L1 expression was not linked to OS, while PD-L1 expression was significantly associated with worse PFS $(\mathrm{HR}=1.98, P=$ 0.003). Further bioinformatics data from available microarray data validated that PD-L1 expression was not correlated with OS $(P>0.1)$ among a larger population (1310 ovarian cancer cases). While PD-L1 expression was significantly associated with unfavorable PFS $(P<$ 0.001 ) in a larger population (1228 patients with ovarian cancer). Although OS is the most common gold standard endpoint. Recent studies have suggested that PFS has become an important and even a primary endpoint in clinical studies. In comparison to OS, PFS may be assessed using shorter and less costly studies [40, 41]. Therefore, the bioinformatics analysis further suggested that PD-L1 was a potential prognostic im-mune marker for ovarian cancer in PFS.

Several potential limitations should be addressed in this systematic review and meta-analysis. First, the search strategy was performed to obtain eligible studies published in English or Chinese. Other studies published in other languages, unpublished papers and conference abstracts were eliminated based on the difficulties in reading. Papers with positive results are more easily published than papers with negative results, which are lacking. These reasons may result in the potential bias and heterogeneity. Second, sample sizes regarding PD-L1 expression with the clinicopathological features of ovarian cancer were also small or absent such as lymph node status or distant metastasis, more studies should be further done in the future. Third, the results might have the potential bias due to the researcher's viewpoint. To diminish this possibility, the study selection and data extraction were performed at least three times to ensure that all data are adequately truthful to the content of all cases included in this meta-analysis. Finally, additional prospective clinical studies should be done to further evaluate the prognostic effect of PD-L1 expression in ovarian cancer, especially for DFS and disease-specific survival.

\section{Conclusions}

In summary, meta-analysis suggested that PD-L1 expression was not linked to tumor grade, clinical stage, lymph node status, tumor histology, OS, and PFS. A bioinformatics study demonstrated that PD-L1 mRNA expression was closely associated with poor PFS, which suggested that PD-L1 may become a promising therapeutic target for PFS of patients with ovarian cancer. To achieve more reliable conclusions, more prospective studies remain needed in the future.

\section{Additional files}

Additional file 1: Figure S1. Publication bias using Egger's test. (TIFF $454 \mathrm{~kb}$ )

Additional file 2: Table S1. Association of PD- $L 1$ expression with the clinicopathological characteristics from TCGA dataset. (DOCX 14 kb)

Human and animal rights

This study was not primary research involving human samples, but our study was a secondary analysis regarding human subject data published in the public domain.

\section{Informed consent}

No patient consent was required because analyses were based on the previously published studies.

\section{Funding}

The authors declare that we have no financial support from any organization.

Availability of data and materials

All data is available in this paper.

\section{Authors' contributions}

LW contributed to the conception and design of this research. LW contributed to the completion of articles, the data extraction, data calculated and design of figures and tables. The author read and approved the final manuscript.

Competing interest

The author declares that he/she has no competing interests. 


\section{Ethics approval and consent to participate}

Although this study was not primary research involving human samples, our study was a secondary analysis regarding human subject data published in the public domain. For this type of study, formal consent is not required.

\section{Consent for publication}

Not applicable.

\section{Publisher's Note}

Springer Nature remains neutral with regard to jurisdictional claims in published maps and institutional affiliations.

\section{Received: 1 December 2018 Accepted: 15 April 2019}

Published online: 30 April 2019

\section{References}

1. Torre LA, Bray F, Siegel RL, Ferlay J, Lortet-Tieulent J, Jemal A. Global cancer statistics, 2012. CA Cancer J Clin. 2015;65(2):87-108. https://doi.org/10.3322/ caac. 21262.

2. Vang R, Shih le M, Kurman RJ. Ovarian low-grade and high-grade serous carcinoma: pathogenesis, clinicopathologic and molecular biologic features, and diagnostic problems. Adv Anat Pathol. 2009;16(5):267-82. https://doi. org/10.1097/PAP.0b013e3181b4fffa.

3. Kaja S, Hilgenberg JD, Collins JL, Shah AA, Wawro D, Zimmerman S, Magnusson R, Koulen P. Detection of novel biomarkers for ovarian cancer with an optical nanotechnology detection system enabling label-free diagnostics. J Biomed Opt. 2012;17(8):081412-1. https://doi.org/10.1117/1. JBO.17.8.081412

4. Cannistra SA. Cancer of the ovary. N Engl J Med. 2004;351(24):2519-29. https://doi.org/10.1056/NEJMra041842.

5. Zou W, Wolchok JD, Chen L. PD-L1 (B7-H1) and PD-1 pathway blockade for cancer therapy: mechanisms, response biomarkers, and combinations. Sci Transl Med. 2016;8(328):328rv324. https://doi.org/10.1126/scitranslmed. aad7118.

6. Agata Y, Kawasaki A, Nishimura H, Ishida Y, Tsubata T, Yagita H, Honjo T. Expression of the PD-1 antigen on the surface of stimulated mouse $T$ and $B$ lymphocytes. Int Immunol. 1996;8(5):765-72.

7. Gato-Canas M, Zuazo M, Arasanz H, Ibanez-Vea M, Lorenzo L, FernandezHinojal G, Vera R, Smerdou C, Martisova E, Arozarena I, Wellbrock C, Llopiz D, Ruiz M, Sarobe P, Breckpot K, Kochan G, Escors D. PDL1 signals through conserved sequence motifs to overcome interferon-mediated cytotoxicity. Cell Rep. 2017;20(8):1818-29. https://doi.org/10.1016/j.celrep.2017.07.075.

8. Pardoll DM. The blockade of immune checkpoints in cancer immunotherapy. Nat Rev Cancer. 2012;12(4):252-64. https://doi.org/10.1038/ nrc3239.

9. Dong H, Strome SE, Salomao DR, Tamura H, Hirano F, Flies DB, Roche PC, Lu J, Zhu G, Tamada K, Lennon VA, Celis E, Chen L. Tumor-associated B7-H1 promotes T-cell apoptosis: a potential mechanism of immune evasion. Nat Med. 2002:8(8):793-800. https://doi.org/10.1038/nm730.

10. Topalian SL, Taube JM, Anders RA, Pardoll DM. Mechanism-driven biomarkers to guide immune checkpoint blockade in cancer therapy. Nat Rev Cancer. 2016;16(5):275-87. https://doi.org/10.1038/nrc.2016.36.

11. Kythreotou A, Siddique A, Mauri FA, Bower M, Pinato DJ. Pd-L1. J Clin Pathol. 2018;71(3):189-94. https://doi.org/10.1136/jclinpath-2017-204853.

12. Hamanishi J, Mandai M, Iwasaki M, Okazaki T, Tanaka Y, Yamaguchi K, Higuchi T, Yagi H, Takakura K, Minato N, Honjo T, Fujii S. Programmed cell death 1 ligand 1 and tumor-infiltrating CD8+ T lymphocytes are prognostic factors of human ovarian cancer. Proc Natl Acad Sci U S A. 2007;104(9): 3360-5. https://doi.org/10.1073/pnas.0611533104.

13. Mills AM, Peres LC, Meiss A, Ring KL, Modesitt SC, Abbott SE, Alberg AJ, Bandera EV, Barnholtz-Sloan J, Bondy ML, Cote ML, Funkhouser E, Moorman PG, Peters ES, Schwartz AG, Terry PD, Wallace K, Schildkraut JM. Targetable immune regulatory molecule expression in high-grade serous ovarian carcinomas in African American women: a study of PD-L1 and IDO in 112 cases from the African American Cancer epidemiology study (AACES). Int Gynecol Pathol. 2018. https://doi.org/10.1097/PGP.0000000000000494.

14. Moher D, Liberati A, Tetzlaff J, Altman DG, Group P. Preferred reporting items for systematic reviews and meta-analyses: the PRISMA statement. PLoS Med. 2009:6(7):e1000097. https://doi.org/10.1371/journal.pmed. 1000097.
15. Coory MD. Comment on: heterogeneity in meta-analysis should be expected and appropriately quantified. Int J Epidemiol. 2010;39(3):932 author reply 933. https://doi.org/10.1093/ije/dyp157.

16. Higgins JP, Thompson SG, Deeks JJ, Altman DG. Measuring inconsistency in meta-analyses. BMJ. 2003;327(7414):557-60. https://doi.org/10.1136/bmj.327. 7414.557.

17. Lau J, loannidis JP, Schmid CH. Quantitative synthesis in systematic reviews. Ann Intern Med. 1997:127(9):820-6.

18. Egger M, Davey Smith G, Schneider M, Minder C. Bias in meta-analysis detected by a simple, graphical test. BMJ. 1997:315(7109):629-34.

19. Zhu X, Zhao L, Lang J. The BRCA1 methylation and PD-L1 expression in sporadic ovarian Cancer. Int J Gynecol Cancer. 2018. https://doi.org/10.1097/ IGC.0000000000001334.

20. Drakes ML, Mehrotra S, Aldulescu M, Potkul RK, Liu Y, Grisoli A, Joyce C, O'Brien TE, Stack MS, Stiff PJ. Stratification of ovarian tumor pathology by expression of programmed cell death-1 (PD-1) and PD-ligand- 1 (PD-L1) in ovarian cancer. J Ovarian Res. 2018;11(1):43. https://doi.org/10.1186/s13048018-0414-z.

21. Li MJ, Li HR, Cheng X, Bi R, Tu XY, Liu F, Chen LH. Clinical significance of targeting drug-based molecular biomarkers expression in ovarian clear cell carcinoma. Zhonghua Fu Chan Ke Za Zhi. 2017:52(12):835-43. https://doi. org/10.3760/cma.j.issn.0529-567x.2017.12.008.

22. Zhu J, Wen $\mathrm{H}, \mathrm{Bi}$ R, Wu Y, Wu X. Prognostic value of programmed deathligand 1 (PD-L1) expression in ovarian clear cell carcinoma. J Gynecol Oncol. 2017;28(6):e77. https://doi.org/10.3802/jgo.2017.28.e77.

23. Wang Q, Lou W, Di W, Wu X. Prognostic value of tumor PD-L1 expression combined with CD8(+) tumor infiltrating lymphocytes in high grade serous ovarian cancer. Int Immunopharmacol. 2017;52:7-14. https://doi.org/10. 1016/j.intimp.2017.08.017.

24. Li M, Li H, Liu F, Bi R, Tu X, Chen L, Ye S, Cheng X. Characterization of ovarian clear cell carcinoma using target drug-based molecular biomarkers: implications for personalized cancer therapy. J Ovarian Res. 2017;10(1):9. https://doi.org/10.1186/s13048-017-0304-9.

25. Chatterjee J, Dai W, Aziz NHA, Teo PY, Wahba J, Phelps DL, Maine CJ, Whilding LM, Dina R, Trevisan G, Flower KJ, George AJT, Ghaem-Maghami S. Clinical use of programmed cell Death-1 and its ligand expression as discriminatory and predictive markers in ovarian Cancer. Clin Cancer Res. 2017;23(13):3453-60. https://doi.org/10.1158/1078-0432.CCR-16-2366.

26. Mesnage SJL, Auguste A, Genestie C, Dunant A, Pain E, Drusch F, Gouy S, Morice $P$, Bentivegna E, Lhomme C, Pautier P, Michels J, Le Formal A, Cheaib B, Adam J, Leary AF. Neoadjuvant chemotherapy (NACT) increases immune infiltration and programmed death-ligand 1 (PD-L1) expression in epithelial ovarian cancer (EOC). Ann Oncol. 2017;28(3):651-7. https://doi.org/ 10.1093/annonc/mdw625.

27. Webb JR, Milne K, Kroeger DR, Nelson BH. PD-L1 expression is associated with tumor-infiltrating $T$ cells and favorable prognosis in high-grade serous ovarian cancer. Gynecol Oncol. 2016;141(2):293-302. https://doi.org/10.1016/ j.ygyno.2016.03.008.

28. Darb-Esfahani S, Kunze CA, Kulbe H, Sehouli J, Wienert S, Lindner J, Budczies J, Bockmayr M, Dietel M, Denkert C, Braicu I, Johrens K. Prognostic impact of programmed cell death-1 (PD-1) and PD-ligand 1 (PD-L1) expression in cancer cells and tumor-infiltrating lymphocytes in ovarian high grade serous carcinoma. Oncotarget. 2016;7(2):1486-99. https://doi.org/10.18632/ oncotarget.6429.

29. Gyorffy B, Lanczky A, Szallasi Z. Implementing an online tool for genomewide validation of survival-associated biomarkers in ovarian-cancer using microarray data from 1287 patients. Endocr Relat Cancer. 2012;19(2):197208. https://doi.org/10.1530/ERC-11-0329.

30. Taube JM, Anders RA, Young GD, Xu H, Sharma R, McMiller TL, Chen S, Klein AP, Pardoll DM, Topalian SL, Chen L. Colocalization of inflammatory response with B7-h1 expression in human melanocytic lesions supports an adaptive resistance mechanism of immune escape. Sci Transl Med. 2012 4(127):127ra137. https://doi.org/10.1126/scitranslmed.3003689.

31. Abiko K, Matsumura N, Hamanishi J, Horikawa N, Murakami R, Yamaguchi K, Yoshioka Y, Baba T, Konishi I, Mandai M. IFN-gamma from lymphocytes induces PD-L1 expression and promotes progression of ovarian cancer. $\mathrm{Br} J$ Cancer. 2015;112(9):1501-9. https://doi.org/10.1038/bjc.2015.101.

32. Chen N, Fang W, Zhan J, Hong S, Tang Y, Kang S, Zhang Y, He X, Zhou T, Qin T, Huang Y, Yi X, Zhang L. Upregulation of PD-L1 by EGFR activation mediates the immune escape in EGFR-driven NSCLC: implication for optional immune targeted therapy for NSCLC patients with EGFR mutation. 
J Thorac Oncol. 2015;10(6):910-23. https://doi.org/10.1097/JTO. 0000000000000500 .

33. Quandt D, Jasinski-Bergner S, Muller U, Schulze B, Seliger B. Synergistic effects of IL-4 and TNFalpha on the induction of B7-H1 in renal cell carcinoma cells inhibiting allogeneic T cell proliferation. J Transl Med. 2014; 12:151. https://doi.org/10.1186/1479-5876-12-151.

34. Kronig H, Kremmler L, Haller B, Englert C, Peschel C, Andreesen R, Blank CU. Interferon-induced programmed death-ligand 1 (PD-L1/B7-H1) expression increases on human acute myeloid leukemia blast cells during treatment. Eur J Haematol. 2014;92(3):195-203. https://doi.org/10.1111/ejh.12228.

35. Sznol M. Blockade of the B7-H1/PD-1 pathway as a basis for combination anticancer therapy. Cancer J. 2014;20(4):290-5. https://doi.org/10.1097/PPO. 0000000000000056.

36. Cha YJ, Kim HR, Lee CY, Cho BC, Shim HS. Clinicopathological and prognostic significance of programmed cell death ligand-1 expression in lung adenocarcinoma and its relationship with p53 status. Lung Cancer. 2016;97:73-80. https://doi.org/10.1016/j.lungcan.2016.05.001.

37. Tomioka N, Azuma M, Ikarashi M, Yamamoto M, Sato M, Watanabe KI, Yamashiro K, Takahashi M. The therapeutic candidate for immune checkpoint inhibitors elucidated by the status of tumor-infiltrating lymphocytes (TILs) and programmed death ligand 1 (PD-L1) expression in triple negative breast cancer (TNBC). Breast Cancer. 2018;25(1):34-42. https://doi.org/10.1007/s12282-017-0781-0.

38. Jung Hl, Jeong D, Ji S, Ahn TS, Bae SH, Chin S, Chung JC, Kim HC, Lee MS, Baek MJ. Overexpression of PD-L1 and PD-L2 is associated with poor prognosis in patients with hepatocellular carcinoma. Cancer Res Treat. 2017; 49(1):246-54. https://doi.org/10.4143/crt.2016.066.

39. Enwere EK, Kornaga EN, Dean M, Koulis TA, Phan T, Kalantarian M, Kobel M, Ghatage P, Magliocco AM, Lees-Miller SP, Doll CM. Expression of PD-L1 and presence of CD8-positive T cells in pre-treatment specimens of locally advanced cervical cancer. Mod Pathol. 2017;30(4):577-86. https://doi.org/10. 1038/modpathol.2016.221.

40. Korn RL, Crowley JJ. Overview: progression-free survival as an endpoint in clinical trials with solid tumors. Clin Cancer Res. 2013;19(10):2607-12. https:// doi.org/10.1158/1078-0432.CCR-12-2934.

41. Dodd LE, Korn EL, Freidlin B, Jaffe CC, Rubinstein LV, Dancey J, Mooney MM Blinded independent central review of progression-free survival in phase III clinical trials: important design element or unnecessary expense? I Clin Oncol. 2008;26(22):3791-6. https://doi.org/10.1200/JCO.2008.16.1711.

Ready to submit your research? Choose BMC and benefit from:

- fast, convenient online submission

- thorough peer review by experienced researchers in your field

- rapid publication on acceptance

- support for research data, including large and complex data types

- gold Open Access which fosters wider collaboration and increased citations

- maximum visibility for your research: over $100 \mathrm{M}$ website views per year

At $\mathrm{BMC}$, research is always in progress.

Learn more biomedcentral.com/submissions 